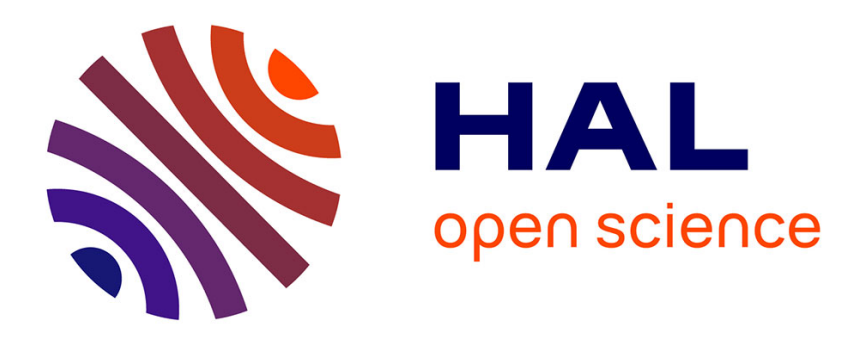

\title{
Recognizing Human Actions based on Extreme Learning Machines
}

\author{
Grégoire Lefebvre, Julien Cumin
}

\section{To cite this version:}

Grégoire Lefebvre, Julien Cumin. Recognizing Human Actions based on Extreme Learning Machines. 11th International Joint Conference on Computer Vision, Imaging and Computer Graphics Theory and Applications, Feb 2016, Roma, Italy. hal-01282009

\section{HAL Id: hal-01282009 \\ https://hal.science/hal-01282009}

Submitted on 3 Mar 2016

HAL is a multi-disciplinary open access archive for the deposit and dissemination of scientific research documents, whether they are published or not. The documents may come from teaching and research institutions in France or abroad, or from public or private research centers.
L'archive ouverte pluridisciplinaire $\mathbf{H A L}$, est destinée au dépôt et à la diffusion de documents scientifiques de niveau recherche, publiés ou non, émanant des établissements d'enseignement et de recherche français ou étrangers, des laboratoires publics ou privés. 


\title{
Recognizing Human Actions based on Extreme Learning Machines
}

\author{
Grégoire Lefebvre and Julien Cumin \\ Orange Labs, $R \& D$, Meylan, France \\ gregoire.lefebvre@orange.com,julien1.cumin@orange.com
}

Keywords: Human Action Recognition, Extreme Learning Machines.

\begin{abstract}
In this paper, we tackle the challenge of action recognition by building robust models from Extreme Learning Machines (ELM). Applying this approach from reduced preprocessed feature vectors on the Microsoft Research Cambridge-12 (MSRC-12) Kinect gesture dataset outperforms the state-of-the-art results with an average correct classification rate of 0.953 over 20 runs, when splitting in two equal subsets for training and testing the 6,244 action instances. This ELM based proposal using a multi-quadric radial basis activation function is compared to other classical classification strategies such as Support Vector Machines (SVM) and Multi-Layer Perceptron (MLP) and advancements are also presented in terms of execution times.
\end{abstract}

\section{INTRODUCTION}

Human activity recognition is one of the main challenging topics in computer vision research. Its importance may be explained by many successes for instance in video games, video surveillance, sport analysis, back and neck pain control, sign language understanding, human-robot interaction, etc. Various types of activities indeed appear in everyday life, such as gestures, actions, human-object interactions, social interactions and group activities.

Nevertheless, some issues still exist in human action recognition automation: the first ones are inherent to user gesture productions, the other ones come from data acquisitions. The classical recognition methods are often biased by numerous factors: dynamical variations (dynamic and phlegmatic users), temporal variations (slow and fast users), physical variations (device weight, user morphology, left or right-handed users, contextual environment, parallel user activities, etc.), paradigm variations (mono versus multi users, open or closed world paradigm, etc.), and cultural interpretation variations (i.e. one gesture may have different meanings regarding several cultures). Other difficulties impacting the data acquisition system are the presence of occlusions, nonrigid motions, view-point changes, background interferences, etc.

Therefore, a human action recognition system is designed to deal with these issues. The early methods propose pose estimators as action features and build appearance models based on shape (Blank et al.,
2005) or motion (Efros et al., 2003) information. Then, in order to avoid segmentation and object tracking issues, local features and bag-of-features representations strategies are proposed. This offers compact action descriptors to be classified as dense trajectories (Wang et al., 2011a) or sparse motion vectors (Kantorov and Laptev, 2014). In contrast, other studiesdeal with mid-level representations (Jain et al., 2013) and temporal models (Amer et al., 2013). On one side, the main objectives are to group point trajectories into tentative action parts by similarity in motion and appearance and then learn discriminative models with latent assignment of action parts. This allows in particular to localize discriminative action parts. On the other side, temporal models describe actions as a sparse sequence of spatio-temporally localized frames. This approach models longer events by temporal logical composition of simple actions.

On action data, preprocessing steps such as spatiotemporal segmentation and description are time consuming. Likewise, parameterizing and training a dedicated classifier can be very challenging. That is why, in this paper, we propose an action classification system based on Extreme Learning Machines (Huang et al., 2004) in order to operate fast and robust recognitions on action data with compact feature vectors.

This paper is organized as follows. Section 2 presents related works on action recognition. In Section 3, we explain in details Extreme Learning Machines (ELM). Then, Section 4 describes our results with a comparison to classical approaches. Finally, our conclusions and perspectives are drawn. 


\section{RELATIVE STUDIES}

Designing an automatic action recognition system based on video analysis is already challenging, but when users want an interactive system based on natural human interactions, the challenge is even higher. Indeed, when designing an iterative action recognition system, the two main priorities are reducing latency when users are interacting with it and maximizing the action recognition scores in order to offer better user experiences. These two properties are influenced by the action feature vector dataset to be modeled in a robustness and speed classifier.

In (Ellis et al., 2013), the two aspects are taken into account with a latency-aware learning formulation in order to train a logistic regression model (LRM). The contribution is to distinguish canonical poses from body motions to reduce ambiguity for action recognition. Their experimental results on the Microsoft Research Cambridge-12 (MSRC-12) Kinect gesture dataset (Fothergill et al., 2012) show improvements (i.e. an average correct classification rate of 0.912 for a 4-cross validation) in opposition to a bags of visual words and a conditional random field strategy. The trade-off between latency and accuracy is then guided by a Frame Based Descriptor (FBD) of size 2276. The information support from the 3D body joint motions appears then to be crucial.

Likewise, in (Hussein et al., 2013), the construction of discriminative descriptors from 3D skeleton data is obtained in order to optimize the recognition system. Encoding the correlation between joint trajectories during space and time, they propose a Temporal Hierarchy of Covariance Descriptor (THCD). This descriptor has a fixed size of 7320 and the final classification is operated by a linear SVM classifier. On the MSRC-12 dataset, the experiments give an average correct classification rate of 0.917 for a 20 -cross validation when splitting $50 \%$ of the dataset for training and testing steps.

A more recent study by (Vemulapalli et al., 2014) also uses a SVM classifier to build an action recognition system. Consequently, it is again the choice of the feature descriptors which influences the global recognition accuracy. The authors propose to model the geometric relationships between 3D body parts. Human actions are then modeled as curves in this Lie group based on 3D rigid body motions which are members of the Special Euclidean Group SE(3). The feature vector is then of size 2280 and the correct classification rate are very challenging with 0.9088 on the Florence3D-Action dataset (Seidenari et al., 2013).

In this paper, we propose a more reduced feature vector of size 1200 and a fast ELM classification.

\section{EXTREME LEARNING MACHINES}

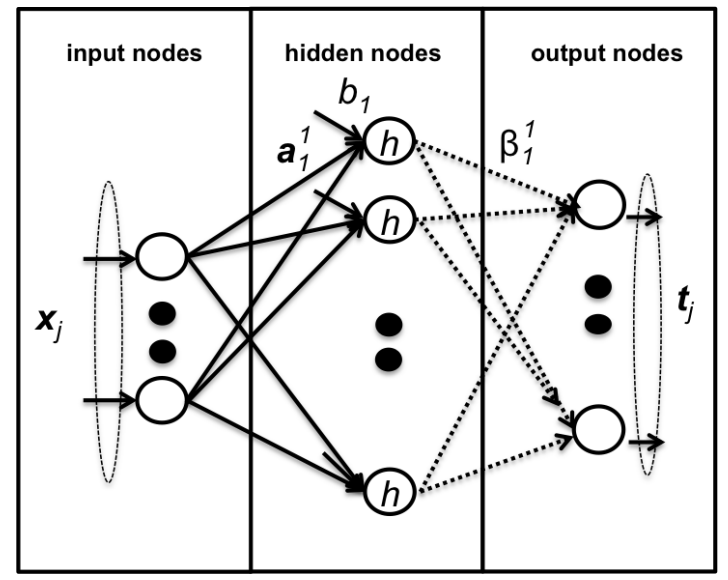

Figure 1: Single feed-forward network.

Deep learning methods are nowadays widely used in machine learning. With recent successes on visual object recognition (Farabet et al., 2013) or speech recognition (Graves et al., 2013) and iconic gesture recognition (Lefebvre et al., 2015), deep architectures using feed-forward neural networks (e.g. Convolutional Neural Networks (Lecun et al., 1998)) or recurrent neural networks (e.g. Long Short Term Memory (Hochreiter and Schmidhuber, 1997)) are limited to some issues: a relatively slow training speed, overfitting problems and a relatively high number of parameters needed to be tuned.

Inspired by previous studies on Radial Basis Function (RBF) networks (see (Broomhead and Lowe, 1988; Chen et al., 1991)), Huang et al. present ELM in (Huang et al., 2004) in order to deal with these issues for classification and regression problems. An ELM is a single layer feed-forward network (SLFN) with only two parameters: the number of hidden nodes and the choice of an activation function (see Figure 1). In comparison to the traditional backpropagation algorithm based on a gradient descent in order to minimize the network error between the produced output and the desired target, the ELM method initializes randomly the network weights and then computes directly a Moore-Penrose pseudo-inverse matrix to generate a unique solution. In various fields (e.g. regression problems, medical diagnosis application, diabetes protein sequence classification), ELM shows some promising features such as generalization ability, robustness, controllability and a fast learning process (see (Huang et al., 2015)).

Numerous studies propose some extensions to address some ELM issues, such as the network hid- 

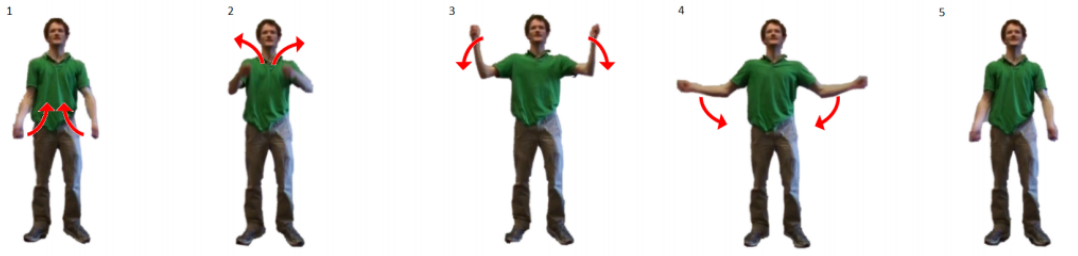

Figure 2: Human action from MSRC-12 Kinect gesture dataset: Wind up the music.

den layer output matrix singularity. In (Wang et al., 2011b), the Effective ELM (EELM) algorithm exploits the strictly dominant criterion for non-singular matrices. In (Huang et al., 2012), an Optimizationbased regularized ELM (ORELM) enhances the generalization properties of ELM. Likewise, improvements are published in (Iosifidis et al., 2014) in order to exploit the training data dispersion with a Minimum Variance ELM (MVELM).

Let's introduce some notations. For $N$ arbitrary distinct samples $\left(\mathbf{x}_{j}, \mathbf{t}_{j}\right)$, we note data samples by $\mathbf{x}_{j}=\left[x_{j}^{1}, x_{j}^{2}, \ldots, x_{j}^{n}\right]^{\top} \in \mathbb{R}^{n}$ and the relative targets by $\mathbf{t}_{j}=\left[t_{j}^{1}, t_{j}^{2}, \ldots, t_{j}^{m}\right]^{\top} \boldsymbol{\top} \in \mathbb{R}^{m}$. Let be $\mathbf{a}_{i}=\left[a_{i}^{1}, a_{i}^{2}, \ldots, a_{i}^{n}\right]^{\top}$ the weight vector connecting the $i^{\text {th }}$ hidden node and the input nodes, and $b_{i} \in \mathbb{R}$ is the respective bias of the $i^{\text {th }}$ hidden node. The weight vector connecting the $i^{\text {th }}$ hidden node and the output nodes is $\beta_{i}=$ $\left[\beta_{i}^{1}, \beta_{i}^{2}, \ldots, \beta_{i}^{m}\right]^{\top}$ and the activation function $h$ is usually a Sigmoid or a Radial Basis Function. Consequently, a SLFN with $L$ hidden neurons is defined by:

$$
\forall j \in\{1, \ldots, n\}, \mathbf{t}_{j}=\sum_{i=1}^{L} \beta_{i} h\left(\mathbf{a}_{i} \cdot \mathbf{x}_{j}+b_{i}\right)
$$

Equation 1 can be written with $H \in \mathbb{R}^{N \times L}$ the hidden layer output matrix of the network, $B \in \mathbb{R}^{L \times m}$ and $T \in \mathbb{R}^{N \times m}$, as defined by:

$$
H B=T .
$$

Therefore, the SFLN learning process corresponds now to find the Equation 2 least squares solution. The solution is provided by:

$$
\hat{B}=H^{\dagger} T \text {. }
$$

In Equation 3, $H^{\dagger}$ represents Moore-Penrose generalized inverse of the hidden layer output matrix $H$. As described in (Zhang et al., 2013), the optimal solution $\hat{B}$ has the following features:

1. this algorithm can gain the minimal training error;

2. this algorithm can get the optimal generalization capability of the minimum paradigm of the output connection weights and network;

3. $\hat{B}$ is unique and can avoid local optimal solutions.
Consequently, the ELM learning speed is fast and can reach the solution straightforward without facing local minima or over-fitting issues. Recent studies (Minhas et al., 2010; Iosifidis et al., 2014) proposed ELM for Human Action Recognition based on bags of visual words. Here, we build our system on specific feature vectors using directly four joint trajectories, described hereafter.

\section{EXPERIMENTS}

\subsection{Protocols}

Our experiments are based on the Microsoft Research Cambridge-12 (MSRC-12) Kinect gesture dataset (see for details (Fothergill et al., 2012) and an action sample in Figure 2). This dataset is composed of 594 sequences of human actions from 30 people performing 12 gestures. The gesture set is composed of 6 iconic gestures: crouch and hide (duck), shoot with a pistol, throw an object, change weapon, kick to attack, put on night vision goggles and 6 metaphoric gestures: start the music (lift outstretched arms), navigate to next menu (push right), wind up the music, take a bow, protest the music, lay down the song tempo (beat both arms). In total, 6, 244 gesture instances are stored using 20 body joint positions captured at $30 \mathrm{~Hz}$. Each body joint is 3-dimensional.

In this study, we evaluate the effectiveness of our approach with a reduced training set using $50 \%$ of the MSRC-12 dataset (i.e. 3,122 training instances and 3,122 test instances). This configuration appears to be the less advantageous and the more challenging one according to (Hussein et al., 2013). In order to compare our results with two state-of-theart methods: Frame-Based Descriptors and Logistic Regression Models (Ellis et al., 2013) (FBD+LRM) and Temporal Hierarchy of Covariance Descriptors and Support Vector Machines (Hussein et al., 2013) (THCD+SVM), we perform a 20-cross validation as in (Hussein et al., 2013) and the comparison is then based on the average correct classification and the associated standard deviation. We evaluate as well two 
other classical classifiers (i.e. SVM and MLP) with the Weka data mining software (Hall et al., 2009) on our feature vectors $(\mathrm{FV})$ to prove the relevance of our $(\mathrm{FV}+\mathrm{ELM})$ approach.

Our feature vectors are obtained from the concatenation of the four 3D body joints corresponding to the two hands and the two feet, for each time period. We first filter the 12-D signal with a low-pass filter of parameter 0.7 . Then a normalization is operated and an interpolation is computed to get an equal duration of $3333 \mathrm{~ms}$ for all actions. The feature vector is finally the concatenation of all values through time, giving a dimension of 1200 . This can be viewed as a prior fusion process.

The choice of all parameters in the following study is given by analyzing a preliminary study with 1200 training instances and 1200 validation instances extracted from the original 3,122 training instances.

For instance, Figure 3 shows some tested configurations for all classifiers. This figure gives the best ELM performance with 400 hidden nodes and a multi-quadric radial basis activation function as opposed to other ELM configurations with sigmoid and hyperbolic tangent activation functions. Likewise, the preliminary results on the good fit of our feature vectors with a SVM classifier give better performances with a RBF kernel with $\gamma=0.005$ and $c=4$, compared to a polynomial kernel. Our evaluation on MLP gives a best configuration with 100 hidden neurons, 600 learning epochs and a learning rate of 0.005 with a hyperbolic tangent as an activation function.

Consequently, the final configurations of the classifiers reported in the next section are the following:

- SVM uses a RBF kernel with $\gamma=0.005$ and $c=4$;

- MLP uses one layer of 100 hidden neurons, 600 learning epochs and a learning rate of 0.005 with a hyperbolic tangent as an activation function;

- ELM uses 400 hidden nodes and a multi-quadric radial basis activation function.

\subsection{Classification Results}

Table 1 outlines the global performances of each human action recognition strategy. Our solution based on our feature vectors FV and an ELM classifier gives the best average correct classification rate of 0.953 with a reduced standard deviation of 0.003 . The results previously published were respectively 0.912 for FBD+LRM (Ellis et al., 2013) (n.b. the authors used only a 4 folds cross-validation) and 0.917 for THCD+SVM (Hussein et al., 2013). Consequently, these results show that feature vectors extracted only
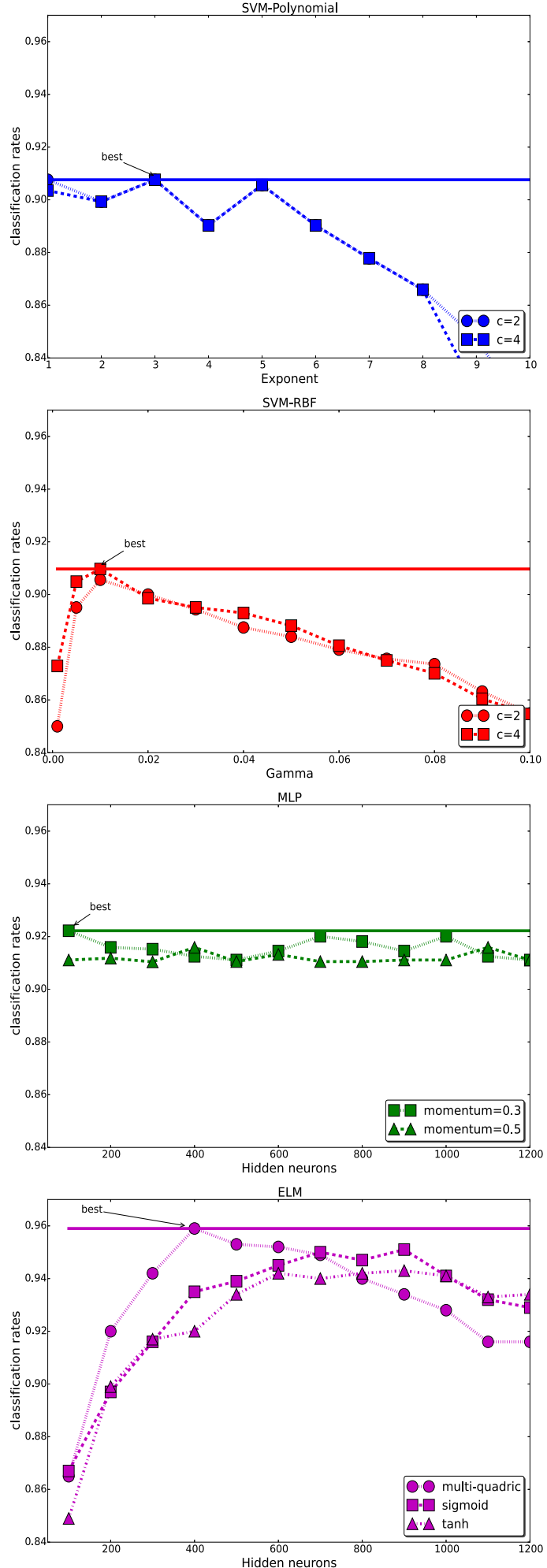

Figure 3: Classification rate on an evaluation dataset (1200 training instances, 1200 evaluation instances). 
Table 1: Average correct Classification Rates (ACR) and Standard Deviations (SD) on MSRC-12.

\begin{tabular}{|c|c|}
\hline Database & MSRC-12 \\
\hline Methods & ACR \& SD \\
\hline \hline $\begin{array}{c}\text { FBD+LRM } \\
\text { (Ellis et al., 2013) }\end{array}$ & $0.912 \pm-$ \\
\hline $\begin{array}{c}\text { THCD+SVM } \\
\text { (Hussein et al., 2013) }\end{array}$ & $0.917 \pm-$ \\
\hline FV+SVM & $0.898 \pm 0.002$ \\
\hline FV+MLP & $0.906 \pm 0.008$ \\
\hline \hline FV+ELM & $\mathbf{0 . 9 5 3} \pm \mathbf{0 . 0 0 3}$ \\
\hline
\end{tabular}

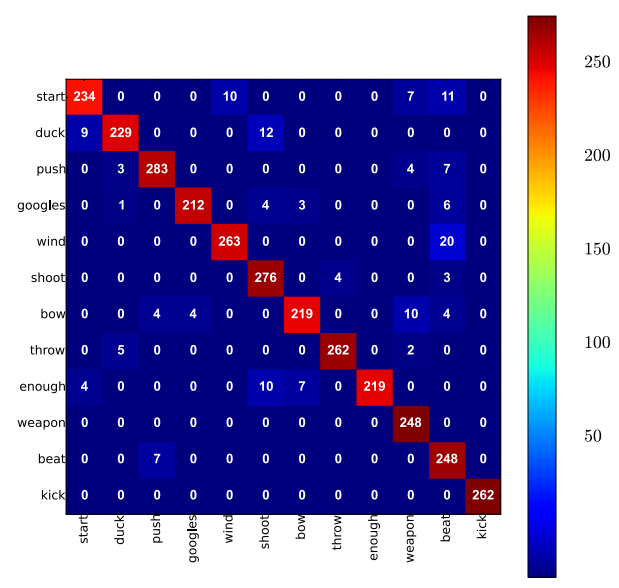

Figure 4: One resulting confusion matrix on MSRC-12, when applying our FV+ELM strategy.

from four joint data can be an adequate information support for human action recognition. Moreover, we demonstrate on our protocol the effectiveness of the ELM classifier as opposed to the SVM and MLP classifiers (with respectively $0.898 \pm 0.002$ and $0.906 \pm 0.008$ of correct classification rate).

A main conclusion of an analysis of confusion matrices (see Figure 4 showing one tested configuration) is that our FV+ELM method presents 20 misclassification between wind up the music (wind) and lay down the tempo (beat). In fact, these two actions share common trajectories with respectively circular movements with both arms, in front of the body, for the first action, and hand beat movements in the air for the second action. Likewise, we can observe some confusions between duck and shoot, start and beat, start and wind up, and enough and shoot.
Table 2: Average Computing Times (ACT) and Standard Deviation (SD) in seconds on MSRC-12.

\begin{tabular}{|c|c|}
\hline Database & MSRC-12 \\
\hline Methods & ACT \& SD \\
\hline \hline FV+SVM & $8.875 \pm 0.182$ \\
\hline FV+MLP & $793.883 \pm 0.194$ \\
\hline \hline FV+ELM & $\mathbf{4 . 8 6 5} \pm \mathbf{0 . 1 0 2}$ \\
\hline
\end{tabular}

\subsection{Computing Times}

In addition, we report the computing times for the 3 methods using the same feature vectors in order to learn 3,122 gestures and testing 3,122 gestures of the MSRC-12 database (c.f. Table 2). This experiment was executed on an Intel Core i5 CPU clocked at 2.67 $\mathrm{GHz}$ with $3.42 \mathrm{~GB}$ of RAM.

These experimental results show that the computing times for the ELM based solution is fast (i.e. around 4.865 seconds in average). On the contrary, the $\mathrm{FV}+\mathrm{SVM}$ based solution requires more time to learn and test the 6,244 gestures (i.e. around 8.875 seconds in average). The FV+MLP based method needs around 793.883 seconds in average but this is mainly due to the learning process using 600 epochs to build the best neural model in this case.

Consequently, our proposed FV+ELM system, achieving the best result performances in a multi-user configuration with a fast recognition computing time, is a very challenging solution.

\section{CONCLUSIONS AND PERSPECTIVES}

In this article, we presented a human action recognition system based on specific feature vectors FV and a ELM classifier. Using the MSRC-12 dataset in order to cover real world challenging cases, we showed that the proposed approach proves to be superior to some state-of-the-art methods. It also outmatches its neural counterpart MLP, both in classification and processing time on our experiments.

Some perspectives would be to experiment Echo State Networks (ESN (Tong et al., 2007)) with raw temporal input data to preserve the temporal correlation and detect gestural grammar inside each human action. 


\section{REFERENCES}

Amer, M., Todorovic, S., Fern, A., and Zhu, S.-C. (2013). Monte carlo tree search for scheduling activity recognition. In Computer Vision (ICCV), 2013 IEEE International Conference on, pages 1353-1360.

Blank, M., Gorelick, L., Shechtman, E., Irani, M., and Basri, R. (2005). Actions as space-time shapes. In Computer Vision, 2005. ICCV 2005. Tenth IEEE International Conference on, volume 2, pages 1395-1402 Vol. 2.

Broomhead, D. S. and Lowe, D. (1988). Multivariable Functional Interpolation and Adaptive Networks. Complex Systems 2, pages 321-355.

Chen, S., Cowan, C., and Grant, P. (1991). Orthogonal least squares learning algorithm for radial basis function networks. Neural Networks, IEEE Transactions on, 2(2):302-309.

Efros, A., Berg, A., Mori, G., and Malik, J. (2003). Recognizing action at a distance. In Computer Vision, 2003. Proceedings. Ninth IEEE International Conference on, pages 726-733 vol.2.

Ellis, C., Masood, S. Z., Tappen, M. F., Laviola, Jr., J. J., and Sukthankar, R. (2013). Exploring the trade-off between accuracy and observational latency in action recognition. Int. J. Comput. Vision, 101(3):420-436.

Farabet, C., Couprie, C., Najman, L., and LeCun, Y. (2013). Learning hierarchical features for scene labeling. Pattern Analysis and Machine Intelligence, IEEE Transactions on, 35(8):1915-1929.

Fothergill, S., Mentis, H. M., Kohli, P., and Nowozin, S. (2012). Instructing people for training gestural interactive systems. In Konstan, J. A., Chi, E. H., and Höök, K., editors, CHI, pages 1737-1746. ACM.

Graves, A., Mohamed, A., and Hinton, G. E. (2013). Speech recognition with deep recurrent neural networks. In IEEE International Conference on Acoustics, Speech and Signal Processing, ICASSP 2013, Vancouver, BC, Canada, May 26-31, 2013, pages 6645-6649.

Hall, M., Frank, E., Holmes, G., Pfahringer, B., Reutemann, P., and Witten, I. H. (2009). The weka data mining software: An update. In SIGKDD Explorations, volume 11.

Hochreiter, S. and Schmidhuber, J. (1997). Long short-term memory. Neural Comput., 9(8):1735-1780.

Huang, G., Huang, G.-B., Song, S., and You, K. (2015). Trends in extreme learning machines: A review. $\mathrm{Neu}$ ral Networks, 61(0):32 - 48 .

Huang, G.-B., Zhou, H., Ding, X., and Zhang, R. (2012). Extreme learning machine for regression and multiclass classification. Systems, Man, and Cybernetics, Part B: Cybernetics, IEEE Transactions on, 42(2):513-529.

Huang, G.-B., Zhu, Q.-Y., and Siew, C.-K. (2004). Extreme learning machine: a new learning scheme of feedforward neural networks. In Neural Networks, 2004. Proceedings. 2004 IEEE International Joint Conference on, volume 2, pages 985-990 vol.2.
Hussein, M. E., Torki, M., Gowayyed, M. A., and El-Saban, M. (2013). Human action recognition using a temporal hierarchy of covariance descriptors on $3 \mathrm{~d}$ joint locations. In Proceedings of the Twenty-Third International Joint Conference on Artificial Intelligence, IJCAI'13, pages 2466-2472. AAAI Press

Iosifidis, A., Tefas, A., and Pitas, I. (2014). Minimum variance extreme learning machine for human action recognition. In Acoustics, Speech and Signal Processing (ICASSP), 2014 IEEE International Conference on, pages 5427-5431.

Jain, A., Gupta, A., Rodriguez, M., and Davis, L. (2013). Representing videos using mid-level discriminative patches. In Computer Vision and Pattern Recognition (CVPR), 2013 IEEE Conference on, pages 25712578.

Kantorov, V. and Laptev, I. (2014). Efficient feature extraction, encoding, and classification for action recognition. In Computer Vision and Pattern Recognition (CVPR), 2014 IEEE Conference on, pages 25932600.

Lecun, Y., Bottou, L., Bengio, Y., and Haffner, P. (1998). Gradient-based learning applied to document recognition. Proceedings of the IEEE, 86(11):2278-2324.

Lefebvre, G., Berlemont, S., Mamalet, F., and Garcia, C. (2015). Inertial gesture recognition with BLSTMRNN. In Koprinkova-Hristova, P., Mladenov, V., and Kasabov, N. K., editors, Artificial Neural Networks, volume 4 of Springer Series in Bio-/Neuroinformatics, pages 393-410. Springer International Publishing.

Minhas, R., Baradarani, A., Seifzadeh, S., and Jonathan Wu, Q. M. (2010). Human action recognition using extreme learning machine based on visual vocabularies. Neurocomput., 73(10-12):1906-1917.

Seidenari, L., Varano, V., Berretti, S., Del Bimbo, A., and Pala, P. (2013). Recognizing actions from depth cameras as weakly aligned multi-part bag-of-poses. In Computer Vision and Pattern Recognition Workshops (CVPRW), 2013 IEEE Conference on, pages 479-485.

Tong, M. H., Bickett, A. D., Christiansen, E. M., and Cottrell, G. W. (2007). Learning grammatical structure with echo state networks. Neural Networks, 20(3):424 - 432. Echo State Networks and Liquid State Machines.

Vemulapalli, R., Arrate, F., and Chellappa, R. (2014). Human action recognition by representing $3 \mathrm{~d}$ skeletons as points in a lie group. In Computer Vision and Pattern Recognition (CVPR), 2014 IEEE Conference on, pages 588-595.

Wang, H., Klaser, A., Schmid, C., and Liu, C.-L. (2011a). Action recognition by dense trajectories. In Computer Vision and Pattern Recognition (CVPR), 2011 IEEE Conference on, pages 3169-3176.

Wang, Y., Cao, F., and Yuan, Y. (2011b). A study on effectiveness of extreme learning machine. Neurocomputing, 74(16):2483-2490.

Zhang, Y., Ding, S., Xu, X., Zhao, H., and Xing, W. (2013). An algorithm research for prediction of extreme learning machines based on rough sets. Journal of Computers, 8(5). 